\title{
Pharmacological effect by the combination of molecular target medicine and interferon gamma
}

\author{
Hiromichi Tsuchiya ${ }^{1}$, Shotaro Kamijyo ${ }^{1}$, Toshihiko Gocho ${ }^{1}$, Yoshitaka Yamazaki ${ }^{2}$, Yui Akita ${ }^{3}$ \\ ${ }^{I}$ Medical pharmacology, Showa university, Japan, ${ }^{2}$ Department of Hospital Pharmaceutics, Showa university, Japan, \\ ${ }^{3}$ School of Pharmacy, Showa university, Japan
}

ErbB2 is amplified in $\sim 30 \%$ of breast cancer patients, and its amplification is associated with poor prognosis and worse survival outcome. We have studied about the antitumor mechanism of Herceptin which is a monoclonal antibody to human ErbB2 receptor. When we used anti ErbB2-neu monoclonal antibody in mouse xenograft model, we found that the number and the activation of interferon gamma positive CD8T cells are changed when the effectiveness of antibody are changed. We found that. We started combined antibody and gamma interferon therapy to mouse xenograft model. Gamma interferon alone showed malignant effects, indicating that immune therapies mediated by this cytokine alone are unlikely to be beneficial. However, we discovered that combined antibody and gamma interferon therapy showed the dramatic synergistical inhibition of tumor growth in vivo MMTV-neu transgenic mouse model. We found an increase of M1 macrophage accumulation in the tumor tissues as well as diminished myeloid derived suppressor cells, and increased CD8T cell cytotoxicity against tumor cells in the antibody and gamma interferon therapy. The antitumor effect was not found when we used the interferon gammaR1 knocked out tumor cell in mouse Xenograft model. So, we discovered that the combined therapy acted on tumor cells directly, led phenotypic changes of the character, for example the tumor stem cell marker ALDH-1, indicating a loss of stem cell like properties, then showed antitumor effect. We used Docetaxel with antibody and interferon gamma. That showed the higher antitumor effect than conventional therapy. From the prevention model, the survival rate was largely improved. That means this therapy is useful as postoperative adjuvant chemotherapy. Also, we showed higher antitumor effect with using PD1 and PDL1 antibody. We showed resistant acquired cells would be treat sensitive to the antibody and interferon gamma therapy. This combined effect was confirmed with Cetuximab and Rituximab. We proved new reactions of monoclonal antibody and interferon gamma. Also, we showed promising possibility of the clinical application of this combined therapy. We believe this finding will help to understand the elucidation of the mechanism of the molecular target treatment and to develop new drugs. 